\title{
Association of mutations in DNA polymerase epsilon with increased CD8+ cell infiltration and prolonged progression-free survival in patients with meningiomas
}

\author{
*John W. Rutland, BA, ${ }^{1}$ Jonathan T. Dullea, MPH, ${ }^{1}$ Corey M. Gill, MD, MS, ${ }^{1}$ Danielle Chaluts, BS, ${ }^{1}$ \\ Daniel Ranti, BS, ${ }^{1}$ Ethan Ellis, BS, ${ }^{2}$ Annie Arrighi-Allisan, BA, ${ }^{1}$ Yayoi Kinoshita, DMD, ${ }^{3}$ \\ Russell B. McBride, PhD, MPH, ${ }^{3,4}$ Joshua Bederson, MD, ${ }^{1}$ Michael Donovan, MD, PhD, ${ }^{3}$ \\ Robert Sebra, PhD, ${ }^{2,5}$ Mary Fowkes, MD, PhD, ${ }^{3}$ Melissa Umphlett, MD, ${ }^{3}$ and Raj K. Shrivastava, MD ${ }^{1}$
}

\begin{abstract}
1Department of Neurosurgery, Icahn School of Medicine at Mount Sinai; ${ }^{2}$ Department of Genetics and Genomic Sciences, Icahn School of Medicine at Mount Sinai; ${ }^{3}$ Department of Pathology, Icahn School of Medicine at Mount Sinai; ${ }^{4}$ The Institute for Translational Epidemiology, Icahn School of Medicine at Mount Sinai, New York, New York; and ${ }^{5}$ Sema4, A Mount Sinai venture, Stamford, Connecticut
\end{abstract}

OBJECTIVE Prior studies have demonstrated a relationship between underlying tumor genetics and lymphocyte infiltration in meningiomas. In this study, the authors aimed to further characterize the relationship between meningioma genomics, CD4+ and CD8+ T-cell infiltration, and oncological outcomes of meningiomas. Understanding specific characteristics of the inflammatory infiltration could have implications for treatment and prognostication.

METHODS Immunohistochemically stained meningioma slides were reviewed to assess the CD4+ and CD8+ cell infiltration burden. The relationship between immune cell infiltration and tumor genomics was then assessed using an adjusted ANOVA model. For a specific gene identified by the ANOVA, the relationship between that mutation and tumor recurrence was assessed using Cox regression.

RESULTS In immunohistochemically stained samples from a subcohort of 25 patients, the mean number of CD4+ cells was 42.2/400x field and the mean number of CD8+ cells was $69.8 / 400 \times$ field. Elevated CD8+ cell infiltration was found to be associated with the presence of a mutation in the gene encoding for DNA polymerase epsilon, POLE (51.6 cells/ hpf in wild-type tumors vs 95.9 cells/hpf in mutant tumors; $p=0.0199)$. In a retrospective cohort of 173 patients, the presence of any mutation in POLE was found to be associated with a $46 \%$ reduction in hazard of progression (HR $0.54,95 \%$ $\mathrm{Cl} 0.311-0.952 ; p=0.033)$. The most frequent mutation was a near-C-terminal nonsense mutation.

CONCLUSIONS A potential association was found between mutant POLE and both an increase in CD8+ cell infiltration and progression-free survival. The predominant mutation was found outside of the known exonuclease hot spot; however, it was still associated with a slight increase in mutational burden, CD8+ cell infiltration, and progression-free survival. Alterations in gene expression, resulting from alterations in POLE, may yield an increased presentation of neoantigens, and, thus, greater CD8+ cell-mediated apoptosis of neoplastic cells. These findings have suggested the utility of checkpoint inhibitors in the treatment of POLE-mutant meningiomas.

https://thejns.org/doi/abs/10.3171/2021.11.FOCUS21592

KEYWORDS meningioma; immune response; POLE; immunotherapy; molecular genetics; DNA sequencing

$\mathrm{T}$ HE World Health Organization classification system for meningiomas is an important predictor of longterm oncological outcomes. Three distinct classes of meningiomas are identified: grade I, benign; grade II, atypical; and grade III, anaplastic. ${ }^{1}$ Generally, lower-grade tumors exhibit favorable oncological outcomes, whereas higher-grade tumors typically have poorer prognoses. However, some patients with high-grade tumors do have long recurrence-free survival. Additionally, certain WHO grade I meningiomas share the recurrence risk of highergrade disease. Marciscano et al. demonstrated an elevated 5 -year recurrence risk for grade I meningiomas when

ABBREVIATIONS FFPE = formalin-fixed, paraffin-embedded; PD-1 = programmed cell death protein 1; PFS = progression-free survival; SNP = single-nucleotide polymorphism; SNV = single-nucleotide variant.

SUBMITTED October 1, 2021. ACCEPTED November 16, 2021

INCLUDE WHEN CITING DOI: 10.3171/2021.11.FOCUS21592.

*J.W.R. and J.T.D. contributed equally to this work. 
atypical features were present on biopsy. ${ }^{2}$ These data suggest that the WHO grade alone does not accurately predict disease course. Understanding the underlying mechanisms driving recurrence could offer better prognostication and elucidate possible mechanisms for intervention.

Recent research has begun to elucidate much of the complex genomic landscape of meningiomas and the relationship of this landscape to oncological outcomes. With a prevalence of $40 \%$ to $60 \%$, the gene that most commonly mutates in meningiomas is the tumor suppressor $N F 2 .{ }^{3}$ Other common mutant genes are AKTI, SMO, PI3KCA, $K L F 4, T R A F 7$, and POLR2A. Much work has been devoted to determining genomic factors that contribute to tumor grade and location. ${ }^{3}$ Comparatively, little work has been focused on the relationship between underlying genomic alterations and oncological outcomes. Using the present data set, a recent study demonstrated a $642 \%$ increase in the hazard of death for patients whose tumors harbored mutations in ARIDIA. ${ }^{4}$ Another study examining expressional profiles demonstrated that loss of the dimerization partner, RB-like, E2F and multivulval class B (DREAM) complex was associated with recurrence after resection. ${ }^{5}$ Moreover, a recent study demonstrated an impressive prediction of recurrence using a multiomic approach; however, it may take some time for this approach to reach wide use given the relative complexity of implementation. ${ }^{6}$ While these studies have begun to characterize outcomes, further exploration of the relationship between tumor genomics and oncological outcomes is needed.

Additional investigations have addressed the interaction of systemic immune modulation and meningioma genetics. One study demonstrated a relationship between the 22/del(22q) genotype and increased macrophage infiltration. ${ }^{7}$ Another recent study demonstrated an increased lymphocytic infiltration in tumors with a higher mutational burden. This study further demonstrated a relationship between NF2 mutations and an elevated presence of scattered lymphocytes. ${ }^{8}$ Recent work has also demonstrated a potential role for immunotherapy in treating a well-selected subset of meningiomas. ${ }^{9-11} \mathrm{~A}$ deeper understanding of the immune microenvironment of meningiomas has implications for the potential of immunotherapy as a novel modality of treatment for meningiomas not amenable to curative surgical intervention.

\section{Methods \\ Study Cohort}

The Mount Sinai human subjects IRB reviewed and approved this study and waived informed consent. The study was deemed in compliance with Health Insurance Portability and Accountability Act of 1996 (HIPAA) guidelines. Through a review of all meningioma resections performed at our institution from 1995 to 2017 (approximately 100 resections per year), we selected 255 patients who had available formalin-fixed, paraffin-embedded (FFPE) tissue with whom we formed a retrospective cohort. A board-certified neuropathologist reviewed the histopathological diagnosis, grade, and purity of each case according to 2016 WHO guidelines (all included meningiomas would be classified the same under the updated 2021 guidelines). ${ }^{12}$ We selected those with WHO grade I and II tumors from this cohort of patients who had $\geq 90$ days of postoperative follow-up. We excluded patients with < 90 days of follow-up to minimize the risk of misclassification of any residual unresected tumor as a recurrence. After exclusion, 184 patients were included for analysis. A further 11 patients were excluded from multivariable analysis due to missing data. Clinical characteristics and recurrence outcomes were collected through a retrospective review of our electronic medical record system.

\section{Next-Generation Targeted Sequencing}

Next-generation targeted sequencing was performed on all 184 tissue samples obtained from the patients who met inclusion criteria. Samples were extracted from the FFPE tissue using Maxwell FFPE Plus DNA Purification Kit (Promega Corp.). Next, AmpliSeq Oncomine Comprehensive research panel versions 2.0 and 3.0 (Thermo Fisher Scientific Inc.) were used to generate DNA libraries, as previously described. ${ }^{13}$ Torrent Suite versions 5.6.0 and 5.0.8 (Thermo Fisher Scientific Inc.) and Ion Reporter versions 5.2, 5.6, and 5.8 (Thermo Fisher Scientific Inc.) were used for sequencing data analysis. This panel was selected for coverage of many genes implicated broadly in human malignancy and specifically in meningioma development. Of note, the following genes that are implicated as drivers of meningiomas are sequenced: $N F 2, A K T 1, S M O$, and $P I 3 K C A$. Unfortunately, the commercial sequencing panel used in this study did not contain the meningioma driver mutations KLF4,TRAF7, and POLR2A. Prior studies have reported findings from this cohort. ${ }^{4,14-17}$

\section{Immunohistochemical Staining for Immune Cell Infiltration}

Twenty-five tumors were randomly selected from our tissue bank. From these FFPE tumors, representative tissue sections were selected. Immunohistochemical studies with antibodies to CD4 (anti-CD4 SP35, Roche Diagnostics), CD8 (anti-CD8 SP57, Roche Diagnostics), and FOXP3 (anti-FOXP3 monoclonal, Abcam plc) were performed using the Discovery Ultra VENTANA platform (Roche Diagnostics). A board-certified neuropathologist (M.U.) reviewed these slides to identify the presence of $\mathrm{CD} 4+, \mathrm{CD} 8+$, and FOXP3+ cells within meningioma tissues. The areas showing the highest infiltrating densities were selected and then manually enumerated at $\times 400$ magnification $\left(0.55 \mathrm{~mm}^{2}\right)$. Enumeration of pathological features in the area of highest density is a commonly used practice within neuropathology. ${ }^{18}$

\section{Statistical Methods}

We examined the representativeness of our immunohistochemically stained subcohort using t-tests and the chi-square test. Patients duplicated in the immunohistochemically stained cohort of 25 patients and survival analysis cohort of 184 patients were excluded from the larger cohort of 184 patients for the comparative analysis. We then performed a 2-way ANOVA with the CD4+ or CD8+ cell count as the outcome of interest, and the presence of mutation, WHO grade, and recurrence status (primary vs recurrent) as the independent variables. This was done for 
TABLE 1. Clinical characteristics of patients with meningiomas

\begin{tabular}{cccc}
\hline & $\begin{array}{c}\text { IHC Stained } \\
\text { Cohort }(\mathrm{n}=25)\end{array}$ & $\begin{array}{c}\text { Whole Cohort } \\
(\mathrm{n}=184)\end{array}$ & p Value* \\
\hline Age, yrs & & & 0.695 \\
\hline Median (Q1, Q3) & $59.8(49.1,68.2)$ & $60.4(49.2,68.2)$ & \\
\hline Range & $23.1-95.7$ & $23.1-85.1$ & \\
\hline Female sex & $14(56.0)$ & $120(65.2)$ & 0.435 \\
\hline WHO grade & & & 0.563 \\
\hline I & $9(36)$ & $50(27.2)$ & \\
\hline II & $16(64)$ & $134(72.8)$ & \\
\hline Occurrence & & & 0.471 \\
\hline Primary & $15(60)$ & $127(69.0)$ & \\
\hline Recurrent & $10(40)$ & $57(31.0)$ & \\
\hline Resection extent & & & \\
\hline Gross-total & $15(60)$ & $108(58.7)$ & \\
\hline Subtotal & $6(24)$ & $65(35.3)$ & \\
\hline Missing & $4(16)$ & $11(6.0)$ & \\
\hline
\end{tabular}

$\mathrm{IHC}=$ immunohistochemically.

* $p$ values represent comparisons between the immunohistochemically stained cohort and the survival analysis cohort. Patients represented in both cohorts were excluded from the survival analysis cohort, leaving 165 patients in that cohort for comparison.

genes with a mutational prevalence $>5 \%$ in the subcohort. The least-squares means cell counts in the mutant versus wild-type groups were calculated.

The mutational landscape of a specific gene of interest $(P O L E)$ was visualized using the R package "mutsneedle" (The R Project). Protein domain information was gathered from InterPro (https://www.ebi.ac.uk/interpro/). ${ }^{19}$ Welch's t-test and the Mann-Whitney U-test were used to compare the mutational burden in POLE-mutant tumors with $P O L E$-wild-type tumors.

We then assessed the relationship between mutations in $P O L E$ and the time to radiographic recurrence after resection. Loss to follow-up and death were considered censoring events at the date of the last stable imaging. All remaining patients were censored at the end of the study period in May 2020. We used Kaplan-Meier analysis to estimate survival stratified by the gene of interest. The hazard of recurrence associated with genomic alterations was estimated using the Cox proportional hazards model adjusted for age at the time of surgery, WHO grade, prior recurrence status, and gross-total resection status. Patients for whom there were missing data were excluded from the regression analysis. A subgroup analysis, restricted to the most common specific single-nucleotide polymorphism (SNP), was also performed. To further assess the effect of POLE, we repeated the analysis with overall survival as the outcome of interest.

All plots were generated and all tests were performed in $\mathrm{R}$ version 4.0.4 (The $\mathrm{R}$ Project). The level of significance was set as alpha $=0.05$.

\section{Results \\ Cohort Characteristics}

We considered 255 patients with meningiomas for inclusion; 19 patients with grade III tumors and 52 patients with $<90$ days of follow-up were excluded. A further 11 patients had missing resection extent data and, thus, were excluded from the multivariable analysis. The characteristics of the patients in the immunohistochemically stained cohort and the whole cohort were similar across age, sex, WHO grade, occurrence, and resection extent (Table 1).

\section{Immunohistochemical Staining Results}

The mean numbers of CD4+ and CD8+ cells present in samples of the 25 immunohistochemically stained tumors were 42.2 and 69.8 , respectively. Only 2 of the meningiomas, both recurrent, demonstrated FOXP3 expression on immunohistochemical staining. The first of these samples, a WHO grade II tumor, demonstrated 6 FOXP3+ cells/ hpf. Similarly, the second sample, a WHO grade I tumor, demonstrated 7 cells/hpf. Of the 103 genes we sequenced, there were no mutational similarities.

The genes most frequently altered, with accompanying immunohistochemical staining results, are shown in Table 2. There was a near-significant, decreased CD4+ immune

TABLE 2. Unadjusted and adjusted mean CD4+ and CD8+ cell count by genetic mutational status

\begin{tabular}{|c|c|c|c|c|c|c|c|}
\hline \multirow[b]{3}{*}{ Gene } & \multirow[b]{3}{*}{ No. } & \multicolumn{3}{|c|}{ CD4+ } & \multicolumn{3}{|c|}{ CD8+ } \\
\hline & & \multicolumn{2}{|c|}{$\begin{array}{c}\text { Multivariate Adjusted Least- } \\
\text { Squares Means Cell Count (SE) }\end{array}$} & \multirow{2}{*}{$\begin{array}{l}\text { Adjusted } \\
\text { p Value }\end{array}$} & \multicolumn{2}{|c|}{$\begin{array}{c}\text { Multivariate Adjusted Least- } \\
\text { Squares Means Cell Count (SE) }\end{array}$} & \multirow{2}{*}{$\begin{array}{l}\text { Adjusted } \\
\text { p Value }\end{array}$} \\
\hline & & Wild-Type & Mutant & & Wild-Type & Mutant & \\
\hline NF2 & 16 & $59.3(13.7)$ & $24.9(10.6)$ & 0.0574 & 76.7 (13.3) & $57.6(10.2)$ & 0.2607 \\
\hline POLE & 7 & $38.5(11.3)$ & $35.3(18)$ & 0.8852 & $51.6(9)$ & $95.9(14.4)$ & 0.0199 \\
\hline ARID1A & 6 & $31.2(10.2)$ & $57.5(17.6)$ & 0.2034 & $65.7(9.6)$ & $61.4(16.7)$ & 0.824 \\
\hline PTCH1 & 5 & $43.6(9.8)$ & $12.2(19.5)$ & 0.1594 & $64.7(9.4)$ & $64.6(18.7)$ & 0.9996 \\
\hline$N F 1$ & 4 & $38.8(10.4)$ & $32.3(22.5)$ & 0.8005 & $66.6(9.5)$ & $56.2(20.5)$ & 0.6559 \\
\hline PALB2 & 4 & $35.9(10.8)$ & $43.9(22.9)$ & 0.7654 & $63.2(9.9)$ & 70 (20.9) & 0.781 \\
\hline SMARCB1 & 3 & $42.2(9.3)$ & $2.5(24.4)$ & 0.1382 & $68.5(8.5)$ & $35.2(22.5)$ & 0.1754 \\
\hline MET & 3 & $34.6(9.3)$ & $66.5(26.4)$ & 0.2561 & 65 (8.8) & $61(24.8)$ & 0.8759 \\
\hline NOTCH3 & 3 & $34.8(9.4)$ & $63.9(26.5)$ & 0.3026 & $64.8(8.8)$ & $63.6(24.8)$ & 0.9633 \\
\hline
\end{tabular}

Boldface type indicates statistical significance. 


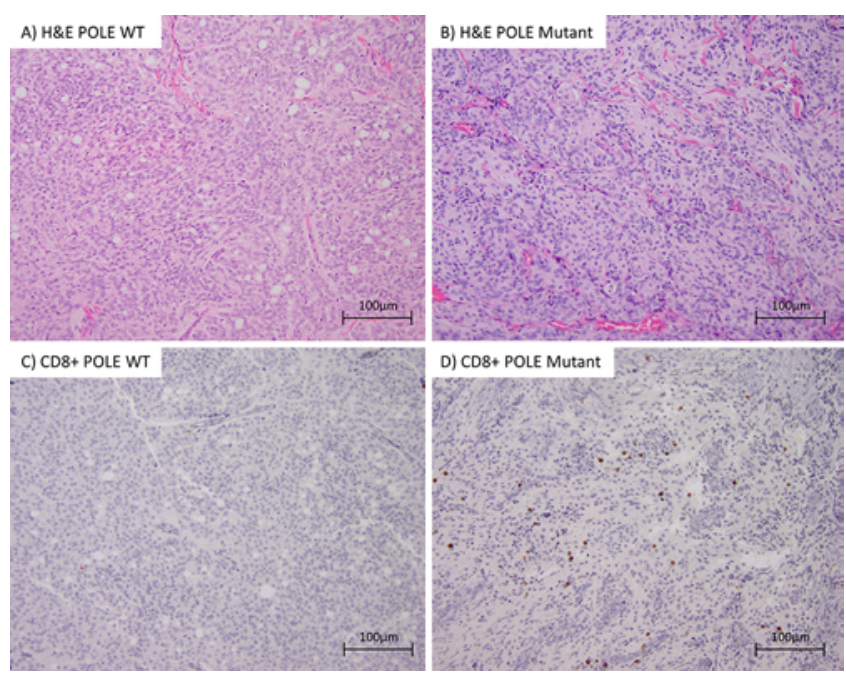

FIG. 1. A and B: Representative H\&E stains of POLE-wild-type and -mutant meningioma tissue. C and D: Representative CD8+ immunohistochemically stained wild-type and mutant meningioma tissue. Original magnification $\times 200$.

cell infiltration for NF2 mutant tumors compared with NF2 wild-type tumors after adjusting for WHO grade and recurrence status (59.3 in wild-type vs 24.9 in mutant tumors; $p=0.057)$. No other significant or near-significant relationships were demonstrated. In looking at the relationship between genomic alterations and CD8+ cell infiltration, a statistically significant relationship was seen between POLE mutation and elevated CD8+ count $(\mathrm{p}=$ 0.0199). After adjusting for WHO grade and recurrence status, the mean number of CD8+ cells in the POLE-mutant tumors was 95.9 (95\% CI 61.1-130.7). After adjusting for the same covariates, the mean number of $\mathrm{CD} 8+$ cells in the POLE-wild-type tumors was 51.6 (95\% CI 29.8-73.4). Figure 1 demonstrates representative H\&Eand anti-CD8-stained microscopic images of 2 grade II meningiomas: 1 with wild-type POLE and 1 with mutant $P O L E$. The number of CD8+ cells stratified across POLE mutational status is shown in Fig. 2 left.

\section{POLE-Mutant Characteristics}

The specific genetic mutations present in the POLEmutant tumors are shown in Fig. 3 and Table 3. Most were nonsense mutations. The most prevalent mutation, Gln2217Ter, was just upstream of a CysB motif important for polymerase epsilon formation. ${ }^{20} P O L E$ is involved in DNA replication and repair; as such, we assessed the number of mutations present, of the genes that we sequenced, in POLE-mutant tumors compared with POLE-wild-type tumors. ${ }^{21}$ The mean number of SNPs in tumors with a mutation in POLE was 13.8 versus 2.3 in POLE-wild-type tumors. The difference of 11.5 mutations is statistically significant $(95 \% \mathrm{CI}$ of difference $=1.9-21.1$ mutations; $\mathrm{p}$ $=0.019)$. The Wilcoxon rank-sum test also demonstrated a similar relationship $(\mathrm{p}<0.0001)$. The number of mutations in the $P O L E$-mutant versus the $P O L E$-wild-type tumors is demonstrated in Fig. 2 right. A similar, yet less dramatic, relationship is demonstrated when restricted to the most common mutant, Gln2217Ter. The mean number of single-nucleotide variants (SNVs) seen in mutant tumors was 4.5 compared with 2.3 in wild-type tumors. A t-test demonstrated that this was a nonsignificant difference $(\mathrm{p}=$ 0.177); however, the Wilcoxon rank-sum test demonstrated a significant difference $(\mathrm{p}<0.001)$.

\section{Survival Analysis}

Given the result of increased CD8+ cell infiltration associated with POLE mutation, a survival analysis was
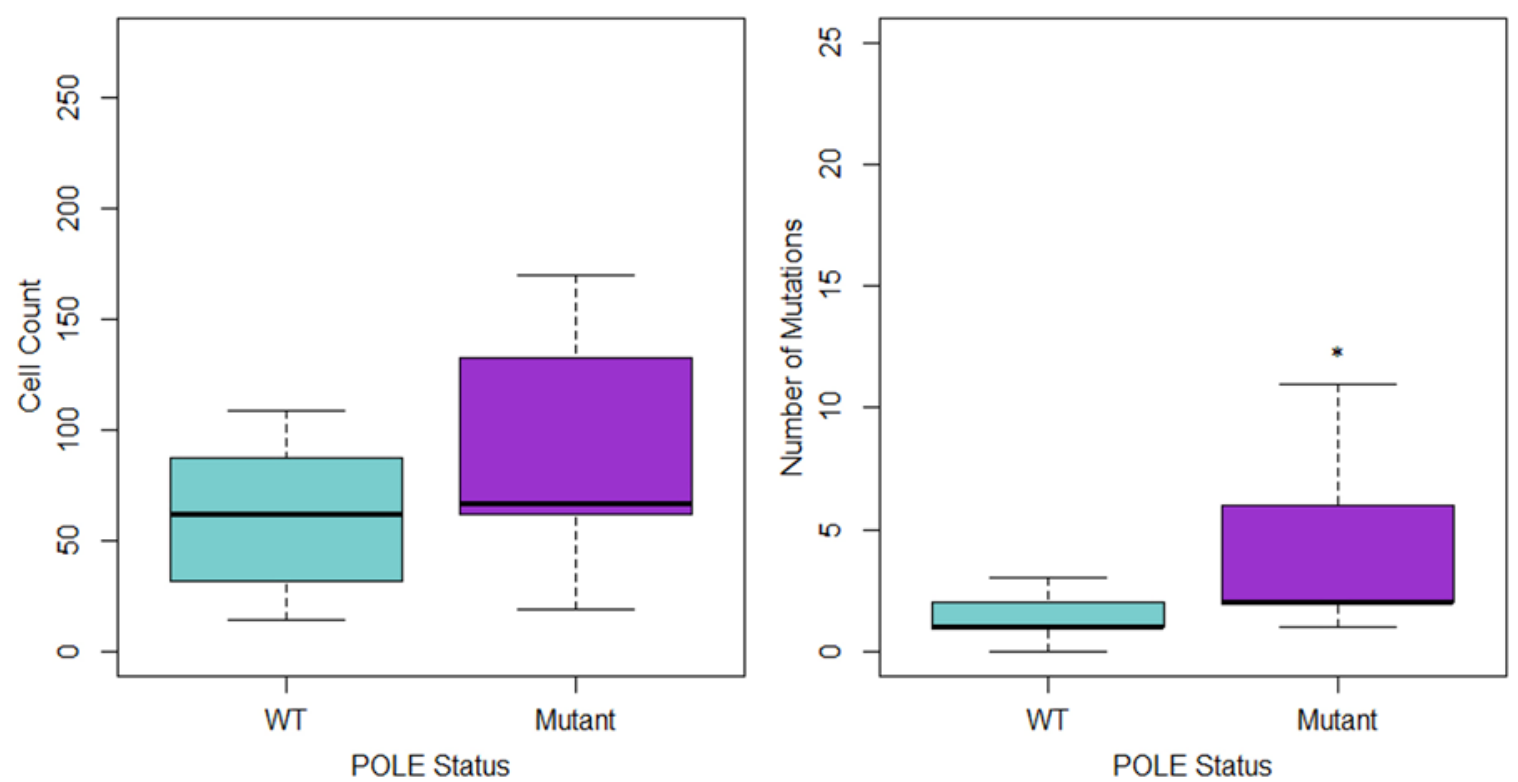

FIG. 2. Left: Boxplot showing the unadjusted CD8+ cell count by POLE mutation status demonstrating increased CD8+ cell burden in POLE-mutant (purple) versus wild-type (blue) tumors. Right: Boxplot showing the number of mutations seen per tumor in the whole cohort with a greater number of mutations in POLE-mutant tumors (purple). *alpha $=0.05$ level of significance. 


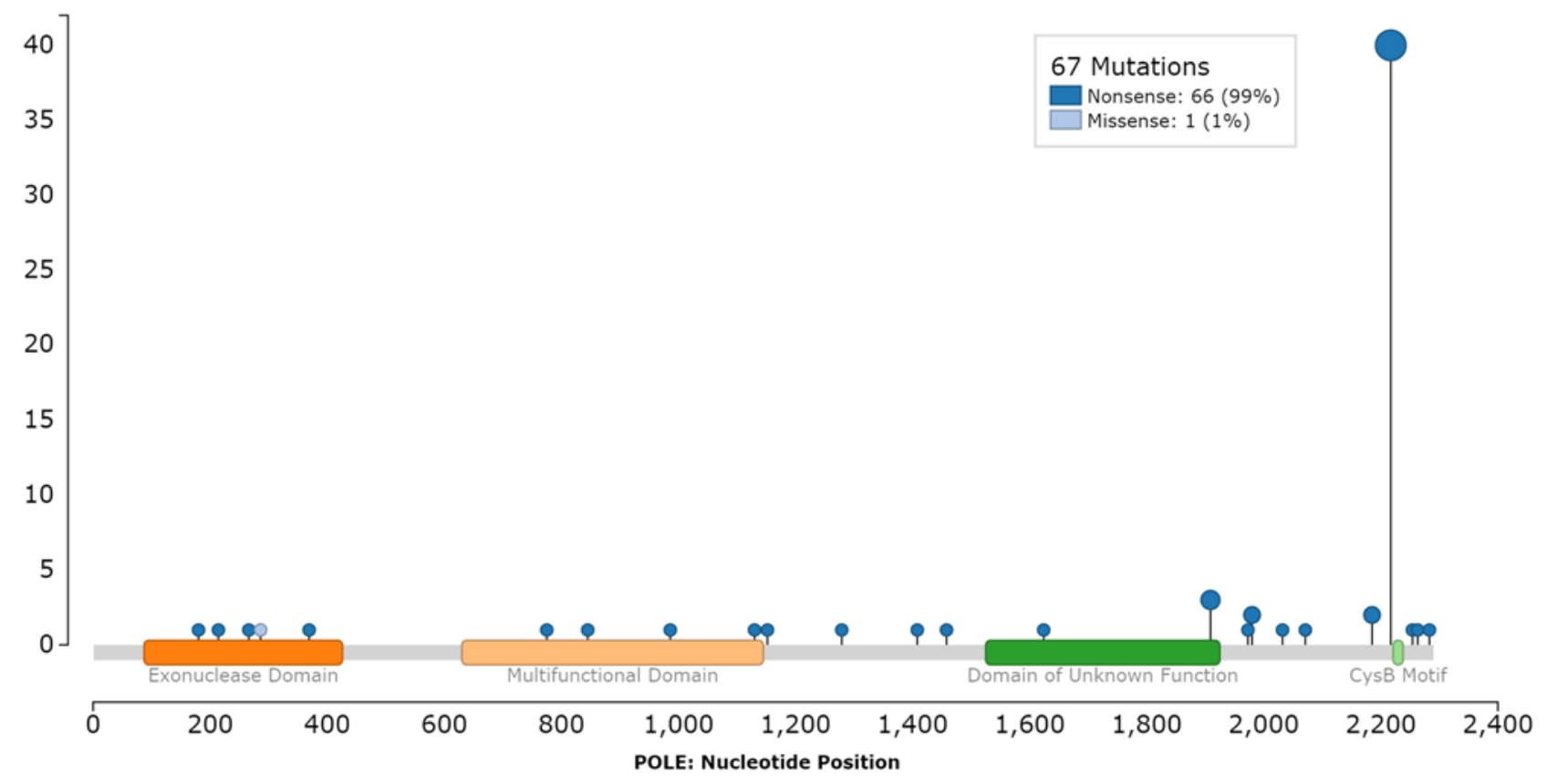

FIG. 3. Lollipop plot of the mutational distribution of POLE mutants included in the study.

performed to assess the relationship between this mutation and overall survival. A Kaplan-Meier analysis was completed in the whole cohort of 184 patients. A total of 82 patients had a recurrence, and the remaining 102 were censored. Kaplan-Meier analysis (Fig. 4) showed a trend toward longer progression-free survival (PFS) in those patients with $P O L E$-mutant tumors than in those with $P O L E$-wild-type tumors. The median recurrence-free survival in patients with $P O L E$-wild-type tumors was 1650 days compared with 2072 days in patients whose tumors harbored POLE mutations. The log-rank test, however, demonstrated that this is not a statistically significant result $(\mathrm{p}=0.13)$. Cox regression analysis adjusting for sex, tumor grade, prior recurrence status, and gross-total resection status was performed in a cohort of 173 patients after excluding those for whom there were missing data (resection extent data were missing for 11 patients). This analysis demonstrated a significant $46 \%$ decreased hazard of recurrence in those patients with a $P O L E$-mutant tumor compared with those who had a POLE-wild-type tumor (HR 0.54, 95\% CI 0.31-0.95; $\mathrm{p}=0.033$ ) (Table 4). To assess the independent contribution of the Gln2071Ter mutation on survival, the hazard ratio was also estimated for the independent effect of this mutation. A Cox regression using the same covariates as above was fit after excluding all non-Gln2217Ter POLE mutants. The relationship held after restricting to this mutant (HR $0.502,95 \%$ CI $0.28-0.91 ; p=0.0231$ ) (Table 4). There was no association between overall survival and a mutation in POLE (HR $0.736,95 \%$ CI 0.271-1.999; $\mathrm{p}=0.5472)($ Table 5).

\section{Discussion}

Herein, we have demonstrated an association between meningiomas with mutant POLE and increased CD8+ lymphocytic infiltration, suggesting that mutations in
TABLE 3. POLE mutation characteristics

\begin{tabular}{ccc}
\hline $\begin{array}{c}\text { Amino Acid } \\
\text { Change }\end{array}$ & $\begin{array}{c}\text { No. of Samples } \\
\text { w/ Mutation }\end{array}$ & $\begin{array}{c}\text { Mean No. of SNVs } \\
\text { per Sample }\end{array}$ \\
\hline p.GIn180Ter & 1 & 2 \\
\hline p.GIn214Ter & 1 & 61 \\
\hline p.Arg266Ter & 1 & 2 \\
\hline p.Pro286Leu & 1 & 2 \\
\hline p.Trp369Ter & 1 & 2 \\
\hline p.Trp775Ter & 1 & 102 \\
\hline p.GIn845Ter & 1 & 22 \\
\hline p.GIn986Ter & 1 & 32 \\
\hline p.Trp1130Ter & 1 & 192 \\
\hline p.GIn1152Ter & 1 & 20 \\
\hline p.Trp1279Ter & 1 & 63 \\
\hline p.GIn1408Ter & 1 & 11 \\
\hline p.Trp1458Ter & 1 & 192 \\
\hline p.Trp1624Ter & 1 & 102 \\
\hline p.Arg1909Ter & 3 & 57.67 \\
\hline p.Glu1973Ter & 1 & 192 \\
\hline p.Trp1980Ter & 2 & 101 \\
\hline p.GIn2032Ter & 1 & 192 \\
\hline p.GIn2071Ter & 1 & 63 \\
\hline p.Trp2185Ter & 2 & 3.5 \\
\hline p.GIn2217Ter & 40 & 102 \\
\hline p.GIn2254Ter & 1 & 192 \\
\hline p.GIn2263Ter & 1 & \\
\hline p.GIn2283Ter & 1 & 102 \\
\hline
\end{tabular}




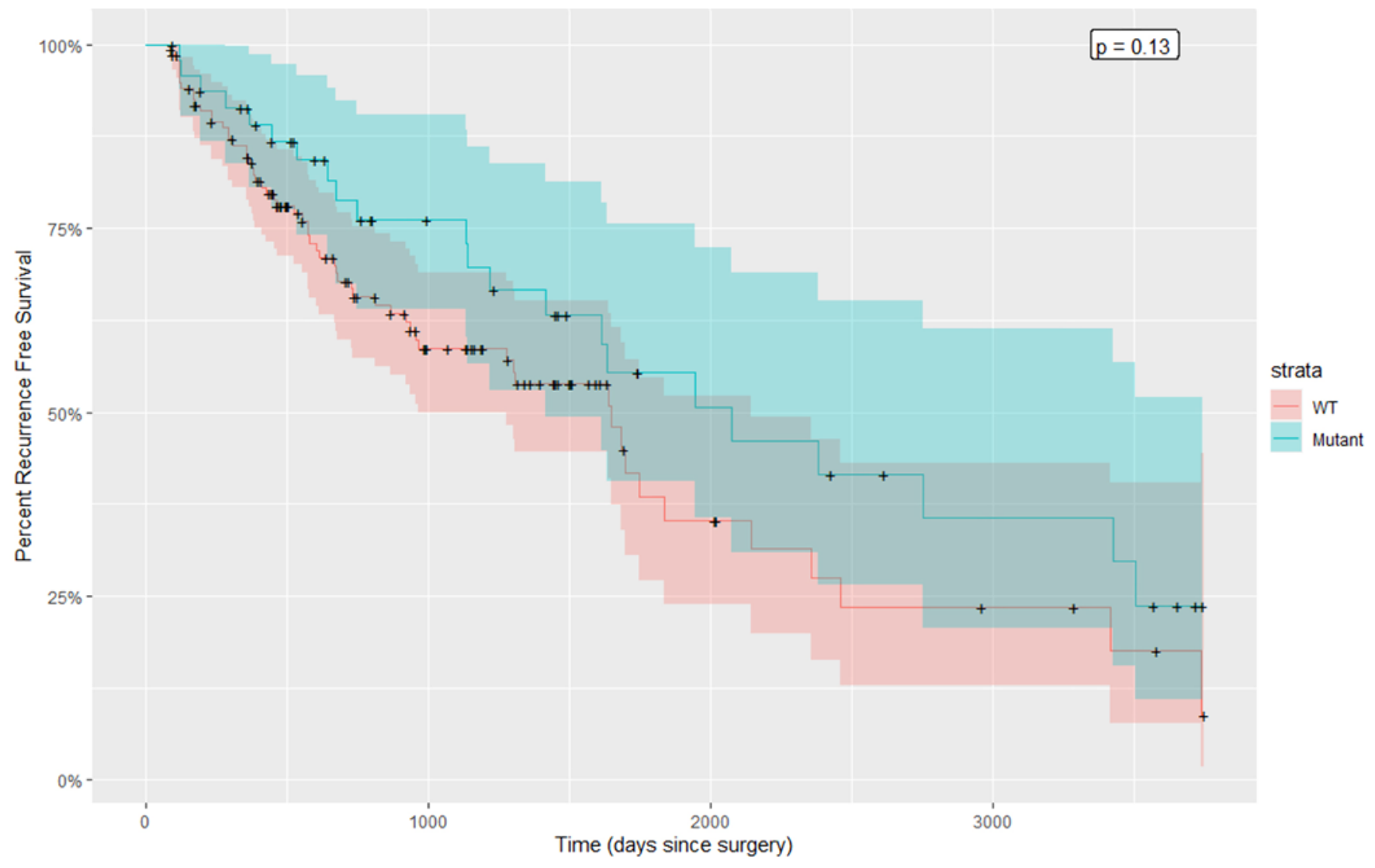

FIG. 4. Kaplan-Meier survival across POLE mutation status demonstrating a trend toward prolonged PFS in POLE-mutant (light blue) tumors compared with wild-type (pink) tumors in the whole cohort.

POLE may be involved in enhanced CD8+ T-cell recruitment. We also demonstrated that the mutations in POLE are associated with an overall increased tumor mutational burden and extended PFS. We did not demonstrate a relationship between POLE and overall survival, which is likely a result of the relatively small number of deaths $(\mathrm{n}=$ 20) observed over the follow-up period.

Studies of other malignancies have linked POLE mutation to lymphocytic infiltration, hypermutant phenotype, and favorable oncological outcomes. ${ }^{22-26}$ However, to date,

TABLE 4. Multivariable Cox regression demonstrated increased PFS in patients with a POLE mutation

\begin{tabular}{|c|c|c|c|c|}
\hline \multirow[b]{2}{*}{ Variable } & \multicolumn{2}{|c|}{ All POLE Mutants $(\mathrm{n}=173)$} & \multicolumn{2}{|c|}{ GIn2071Ter POLE Mutants Only $(\mathrm{n}=166)$} \\
\hline & $\mathrm{HR}(95 \% \mathrm{Cl})$ & p Value & $\mathrm{HR}(95 \% \mathrm{Cl})$ & p Value \\
\hline Age at op & $1.023(1.003-1.043)$ & 0.027 & $1.021(1-1.042)$ & 0.046 \\
\hline \multicolumn{5}{|l|}{ WHO grade } \\
\hline 1 & Referent & & Referent & \\
\hline II & $1.456(0.835-2.538)$ & 0.185 & $1.39(0.789-2.45)$ & 0.254 \\
\hline \multicolumn{5}{|c|}{ Recurrence status } \\
\hline Primary & Referent & & Referent & \\
\hline Recurrent & $2.934(1.765-4.88)$ & $<0.001$ & $2.983(1.768-5.033)$ & $<0.001$ \\
\hline \multicolumn{5}{|c|}{ Resection extent } \\
\hline Gross-total & Referent & & Referent & \\
\hline Subtotal & $1.800(1.097-2.954)$ & 0.020 & $1.768(1.066-2.931)$ & 0.027 \\
\hline \multicolumn{5}{|l|}{ POLE mutation } \\
\hline Wild-type & Referent & & Referent & \\
\hline Mutant & $0.544(0.311-0.952)$ & 0.033 & $0.502(0.277-0.91)$ & 0.023 \\
\hline
\end{tabular}


all these studies have reported mutational hot spots in an exonuclease domain near the N-terminus. This loss of function interferes with the DNA repair process and results in a hypermutant phenotype. ${ }^{27}$ In endometrial cancer, researchers demonstrated an increased infiltration of lymphocytes in POLE-mutant tumors. Further work has characterized this infiltration as CD8+ in nature and protective..$^{22,23}$ A recent study in colorectal cancer demonstrated a similar pattern of increased CD8+ cell infiltration associated with POLE mutation and improved survival outcomes. ${ }^{28}$ This relationship has also been recently demonstrated in the CNS pathology of high-grade gliomas. ${ }^{25}$ These studies have proposed a protective effect of POLE mutation; this protective effect is postulated to be mediated by cytotoxic T-cell control of POLE-mutant neoplastic cells resulting from a greater presentation of neoantigens. ${ }^{22-26,28}$ This provides the theoretical framework on which our hypothesis of a similar immunologically mediated mechanism is based.

In the present study, the most common mutations in the POLE gene were nonsense mutations immediately upstream of the C-terminal adjacent CysB motif. The C-terminal CysB motif is necessary for proper DNA polymerase epsilon formation and folding. ${ }^{20}$ Even with the alterations mostly distant from the exonuclease site, we still saw an increase in the number of mutations for the POLE-mutant tumors with our targeted sequencing; when restricted to the most common mutation, we saw a somewhat attenuated increase in the number of SNVs present. The elevation in mutational burden resulting from alteration to the C-terminus is supported by preclinical evidence. The C-terminus is a location of accessory protein binding. In Saccharomyces cerevisiae, mutations in the protein that binds to the C-terminal portion of Pol2 (S. cerevisiae Pol2 is homologous to human POLE) result in a higher overall mutational burden. It is thought that the resultant destabilized enzyme complex indirectly causes mutagenesis through replication fork destabilization. This destabilization results in replication occurring via less accurate polymerases, such as polymerase delta. ${ }^{29} \mathrm{~A}$ possible extension is that mutations in POLE, which similarly alter the protein-protein interaction, may also increase the mutational burden. This may be the functional consequence of the C-terminal POLE mutation.

Using these preliminary data, we hypothesize that mutations in POLE may result in changes of the expression profile of meningiomas resulting from the potentially altered DNA replication process. Similar to exonuclease domain POLE-mutant tumors, this may yield the potential for enhanced presentation of immunogenic neoantigens. We further contend that this could result in an upregulation of cytotoxic T-cell effector response and, thus, an overall augmented antitumor cytotoxic immune response. This proposed mechanism, while similar to previously theorized mechanisms for POLE-mediated protective effects, is distinct due to the likely weaker effect of the Cterminal mutation on the overall mutational burden.

In patients with POLE-mutant tumors, the presence of an antitumor response is further supported by the prolonged PFS, herein demonstrated for patients who had mutations in POLE. We hypothesize that the enhanced
TABLE 5. Multivariable Cox regression demonstrated no relationship between overall survival and POLE mutation

\begin{tabular}{ccc}
\hline & \multicolumn{2}{c}{ All POLE Mutants $(\mathrm{n}=173)$} \\
\cline { 2 - 3 } Variable & $\mathrm{HR}(95 \% \mathrm{Cl})$ & $\mathrm{p}$ Value \\
\hline Age at op & $1.062(1.02-1.105)$ & 0.0037 \\
\hline WHO grade & \\
\hline $\mathrm{I}$ & Referent & \\
\hline $\mathrm{I}$ & $1.498(0.506-4.437)$ & 0.4657 \\
\hline Recurrence status & & \\
\hline Primary & Referent \\
\hline Recurrent & $2.445(0.919-6.505)$ & 0.0735 \\
\hline Resection extent & & \\
\hline Gross-total & Referent & 0.8138 \\
\hline Subtotal & $1.124(0.424-2.983)$ & \\
\hline POLE mutation & & 0.5472 \\
\hline Wild-type & Referent \\
\hline Mutant & $0.736(0.271-1.999)$ &
\end{tabular}

Boldface type indicates statistical significance.

cytotoxic T-cell presence may partly explain the favorable oncological outcomes of these specific patients. The potential increased immunogenicity of the POLE-mutant tumors could potentially result in a greater degree of immunological control over the growth and progression of residual tumor tissue after resection. Through its effect as a protective molecular genetic event, mutations in POLE could be an important mechanism by which the immune microenvironment is formed in certain meningiomas.

The therapeutic implications behind these findings also warrant discussion. Cancers containing greater mutational burdens have been shown to derive significant clinical benefit from immune checkpoint inhibitors. ${ }^{30-32}$ These findings may be helpful in the identification of a subset of patients with meningiomas that will show a positive therapeutic response to specific immunotherapies such as cytotoxic Tlymphocyte associated protein 4 (CTLA4), programmed cell death protein 1 (PD-1), and programmed death-ligand 1 (PD-L1) inhibitors. Specifically, PD-1 inhibitors have shown some promise in the treatment of meningiomas. A 2017 case report of a patient with co-occurring lung cancer treated with an anti-PD-1 monoclonal antibody demonstrated a reduction in meningioma size. 9 A case report from 2018, published as part of a clinical trial (registration no. NCT02648997, ClinicalTrials.gov), demonstrated a robust response to anti-PD-1 monoclonal antibody in a tumor with a mismatch repair pathway-deficient gene. Interestingly, this tumor also harbored a POLE mutation. ${ }^{33}$ The results from this clinical trial (registration no. NCT02648997, ClinicalTrials.gov) of 25 patients with recurrent WHO grade II and III tumors were recently published; unfortunately, there was not a robust antitumor response across all patients. The mismatch repair tumor, previously reported, was the only tumor of the 25 to have a sustained antitumor response. ${ }^{11}$ While this study may not support the broad utility of anti-PD-1 immunotherapy in meningiomas, it does underscore the importance of the proper selection of tumors for immunotherapy. The POLE mutation may be an 
interesting genetic marker of immune system control. Further work is warranted to determine its utility in identifying patients with meningiomas that can be treated with checkpoint inhibitors to potentiate recurrence-free survival.

Our findings also inform the future basis of tumor grading and classification. Given the limitations of WHO grading, there has been an increased importance placed on molecular markers when determining a specific diagnosis and prognosis. ${ }^{3}$ The most recent update to this classification system has begun to include molecular genomics and epigenetics into the classification system..$^{12}$ Additional tumor molecular markers, such as the POLE mutation, can be used to explain further variations within each grading category, such as inconsistencies in tumor recurrence and survival. Thus, the addition of a further genetic basis of classification provides essential prognostic data within the traditional diagnostic categories determined by histology. The findings in this study and others can ultimately improve diagnostic accuracy, patient management, and treatment response due to targeted therapies.

The strengths of this work are, in part, derived from the linkage between long-term clinical outcomes, genetic sequencing, and lymphocytic infiltration in a large cohort of patients. With this, we were able to identify a relationship between CD8+ cell infiltration, a specific genetic mutation, and prolonged PFS. Furthermore, we were able to propose a mechanism for this improved oncological outcome.

A significant limitation in our present work is the cohort size of immunohistochemically stained samples. While sufficient to identify the relationship between POLE and CD8+ cells, it is underpowered to discover more subtle relationships between other genetic mutational phenotypes and lymphocytic infiltration. We plan to expand upon this work to characterize more subtle, but potentially important, relationships in future investigations. Another related limitation is the presence of multiple comparisons in the ANOVA with the use of a potentially permissive $p$ value. In this exploratory study, we accepted the resultant increased chance of a type I error in favor of limiting the chance of a type II error. We are, however, confident in reporting these results given the biological mechanisms for increased CD8+ infiltration and prolonged survival. Moreover, our sample size was too small to determine a potential relationship between FOXP3+ cell infiltration, genetics, and survival. This sample size restriction resulting in limited information on FOXP3+ cell infiltration was compounded by our restriction to WHO grade I and II meningiomas. Inclusion of grade III meningiomas would likely result in a more successful exploration of the nature of FOXP3+ cells; these are cells that have been shown to be enriched in grade III meningiomas. ${ }^{34}$ In the future, we plan to assess the relationship between FOXP3+ cells and tumor genetics in a cohort of patients with high-grade meningiomas. Another limitation of the present work is the lack of sequencing data for KLF4, TRAF7, or POLR2A; future exploration of the relationship between alterations in these genes and immune cell infiltration is warranted. Given the importance of copy number variation in the development of meningiomas, future work is also warranted to explore the effect of this phenomenon on the immunological microenvironment. A last limitation is the lack of data on radiotherapy, which would likely increase the precision of our results; however, given that it is not likely to be correlated with POLE mutation, confounding is unlikely. Future work that addresses some of these limitations will likely be successful in further elucidating the relationship between meningioma genetics, immune infiltration, and survival outcomes.

\section{Conclusions}

While a favorable oncological outcome can often be obtained with current meningioma treatment paradigms, there remains a subset of patients for whom tumors recur. In this first study to examine the relationship between meningioma mutations and specific CD4+ and CD8+ cell infiltration, we found an increased degree of cytotoxic T-cell infiltration into meningiomas when those meningiomas harbored a mutation in a DNA repair polymerase (polymerase epsilon). This mutation was also associated with an increased mutational burden. Furthermore, the POLE mutation was found to have clinical significance in the form of prolonged PFS for those patients with this mutation in their resected meningioma. The presence of this genotype with enhanced lymphocytic infiltration suggests the possibility of future primary and adjuvant meningioma treatment with immunotherapy.

\section{Acknowledgments}

John W. Rutland was supported by the Rare Disease Foundation and BC Children's Hospital Foundation.

This study was jointly funded by the Rare Disease Foundation and BC Children's Hospital Foundation, and by internal funding at the Icahn School of Medicine at Mount Sinai. John W. Rutland secured the funding from the Rare Disease Foundation.

\section{References}

1. Louis DN, Perry A, Reifenberger G, et al. The 2016 World Health Organization Classification of Tumors of the Central Nervous System: a summary. Acta Neuropathol. 2016;131(6): 803-820.

2. Marciscano AE, Stemmer-Rachamimov AO, Niemierko A, et al. Benign meningiomas (WHO Grade I) with atypical histological features: correlation of histopathological features with clinical outcomes. J Neurosurg. 2016;124(1):106-114.

3. Karsy M, Azab MA, Abou-Al-Shaar H, et al. Clinical potential of meningioma genomic insights: a practical review for neurosurgeons. Neurosurg Focus. 2018;44(6):E10.

4. Gill CM, Loewenstern J, Rutland JW, et al. SWI/SNF chromatin remodeling complex alterations in meningioma. $J$ Cancer Res Clin Oncol. 2021;147(11):3431-3440.

5. Patel AJ, Wan YW, Al-Ouran R, et al. Molecular profiling predicts meningioma recurrence and reveals loss of DREAM complex repression in aggressive tumors. Proc Natl Acad Sci U S A. 2019;116(43):21715-21726.

6. Nassiri F, Liu J, Patil V, et al. A clinically applicable integrative molecular classification of meningiomas. Nature. 2021; 597(7874):119-125.

7. Domingues PH, Teodósio C, Otero Á, et al. Association between inflammatory infiltrates and isolated monosomy 22/ del(22q) in meningiomas. PLoS One. 2013;8(10):e74798.

8. Rutland JW, Gill CM, Loewenstern J, et al. NF2 mutation status and tumor mutational burden correlate with immune cell infiltration in meningiomas. Cancer Immunol Immunother. 2021;70(1):169-176. 
9. Gelerstein E, Berger A, Jonas-Kimchi T, et al. Regression of intracranial meningioma following treatment with nivolumab: case report and review of the literature. J Clin Neurosci. 2017;37:51-53.

10. Dunn IF, Du Z, Touat M, et al. Mismatch repair deficiency in high-grade meningioma: a rare but recurrent event associated with dramatic immune activation and clinical response to PD-1 blockade. JCO Precis Oncol. 2018;2018(2):1-12.

11. Bi WL, Nayak L, Meredith DM, et al. Activity of PD-1 blockade with Nivolumab among patients with recurrent atypical/ anaplastic meningioma: Phase II trial results. Neuro Oncol. Published online May 20, 2021. doi:10.1093/neuonc/noab118

12. Louis DN, Perry A, Wesseling P, et al. The 2021 WHO Classification of Tumors of the Central Nervous System: a summary. Neuro Oncol. 2021;23(8):1231-1251.

13. Pain M, Wang H, Lee E, et al. Treatment-associated TP53 DNA-binding domain missense mutations in the pathogenesis of secondary gliosarcoma. Oncotarget. 2017;9(2):2603-2621.

14. Loewenstern J, Rutland J, Gill C, et al. Comparative genomic analysis of driver mutations in matched primary and recurrent meningiomas. Oncotarget. 2019;10(37):3506-3517.

15. Gill CM, Loewenstern J, Rutland JW, et al. Recurrent IDH mutations in high-grade meningioma. Neuro Oncol. 2020; 22(7):1044-1045.

16. Gill CM, Loewenstern J, Rutland JW, et al. In Reply: Retention of ATRX and DAXX expression in meningiomas. Neurosurgery. 2020;86(2):E244-E246.

17. Gill CM, Loewenstern J, Rutland JW, et al. STK11 mutation status is associated with decreased survival in meningiomas. Neurol Sci. 2020;41(9):2585-2589.

18. Olar A, Wani KM, Sulman EP, et al. Mitotic index is an independent predictor of recurrence-free survival in meningioma. Brain Pathol. 2015;25(3):266-275.

19. D3DXI9 (D3DXI9) - protein. InterPro. Accessed December 9 , 2021. https://www.ebi.ac.uk/interpro/protein/UniProt/D3DXI9/

20. Netz DJA, Stith CM, Stümpfig M, et al. Eukaryotic DNA polymerases require an iron-sulfur cluster for the formation of active complexes. Nat Chem Biol. 2011;8(1):125-132.

21. Blum M, Chang HY, Chuguransky S, et al. The InterPro protein families and domains database: 20 years on. Nucleic Acids Res. 2021;49(D1):D344-D354.

22. van Gool IC, Eggink FA, Freeman-Mills L, et al. POLE proofreading mutations elicit an antitumor immune response in endometrial cancer. Clin Cancer Res. 2015;21(14):3347-3355.

23. Kondratiev S, Sabo E, Yakirevich E, Lavie O, Resnick MB. Intratumoral CD8+ T lymphocytes as a prognostic factor of survival in endometrial carcinoma. Clin Cancer Res. 2004; 10(13):4450-4456.

24. Hussein YR, Weigelt B, Levine DA, et al. Clinicopathological analysis of endometrial carcinomas harboring somatic POLE exonuclease domain mutations. Mod Pathol. 2015; 28(4):505-514.

25. Erson-Omay EZ, Çağlayan AO, Schultz N, et al. Somatic POLE mutations cause an ultramutated giant cell high-grade glioma subtype with better prognosis. Neuro Oncol. 2015; 17(10):1356-1364.

26. Bindea G, Mlecnik B, Tosolini M, et al. Spatiotemporal dynamics of intratumoral immune cells reveal the immune landscape in human cancer. Immunity. 2013;39(4):782-795.
27. Lange SS, Takata K, Wood RD. DNA polymerases and cancer. Nat Rev Cancer. 2011;11(2):96-110.

28. Glaire MA, Domingo E, Sveen A, et al. Tumour-infiltrating $\mathrm{CD} 8^{+}$lymphocytes and colorectal cancer recurrence by tumour and nodal stage. Br J Cancer. 2019;121(6):474-482.

29. Kraszewska J, Garbacz M, Jonczyk P, Fijalkowska IJ, Jaszczur M. Defect of Dpb2p, a noncatalytic subunit of DNA polymerase $\varepsilon$, promotes error prone replication of undamaged chromosomal DNA in Saccharomyces cerevisiae. Mutat Res. 2012;737(1-2):34-42.

30. Łuksza M, Riaz N, Makarov V, et al. A neoantigen fitness model predicts tumour response to checkpoint blockade immunotherapy. Nature. 2017;551(7681):517-520.

31. Van Allen EM, Miao D, Schilling B, et al. Genomic correlates of response to CTLA-4 blockade in metastatic melanoma. Science. 2015;350(6257):207-211.

32. Snyder A, Makarov V, Merghoub T, et al. Genetic basis for clinical response to CTLA-4 blockade in melanoma. $N$ Engl J Med. 2014;371(23):2189-2199.

33. Bielska AA, Chatila WK, Walch H, et al. Tumor mutational burden and mismatch repair deficiency discordance as a mechanism of immunotherapy resistance. J Natl Compr Canc Netw. 2021;19(2):130-133.

34. Du Z, Abedalthagafi M, Aizer AA, et al. Increased expression of the immune modulatory molecule PD-L1 (CD274) in anaplastic meningioma. Oncotarget. 2015;6(7):4704-4716.

\section{Disclosures}

Dr. Bederson owns equity in Surgical Theater, LLC, manufacturer of the Surgical Navigation Advanced Platform (SNAP) system that may be used for intraoperative image guidance in the study.

\section{Author Contributions}

Conception and design: Shrivastava, Bederson, Sebra, Fowkes, Umphlett. Acquisition of data: Gill, Ellis, Umphlett. Analysis and interpretation of data: Shrivastava, Rutland, Dullea, Ranti, Ellis, Arrighi-Allisan, Kinoshita, McBride, Bederson, Donovan. Drafting the article: Rutland, Dullea, Gill, Chaluts, Sebra. Critically revising the article: Shrivastava, Rutland, Dullea, Gill, Chaluts, Arrighi-Allisan, Kinoshita, McBride, Bederson, Donovan, Sebra, Umphlett. Reviewed submitted version of manuscript: Shrivastava, Rutland, Dullea, Gill, Chaluts, Ranti, Ellis, Bederson, Donovan, Sebra, Umphlett. Statistical analysis: Dullea. Study supervision: Shrivastava.

\section{Supplemental Information \\ Videos}

Video Abstract. https://vimeo.com/664714521.

\section{Previous Presentations}

Portions of this work were presented in poster form at the 2021 CNS Annual Meeting, Austin, Texas, October 19, 2021.

\section{Correspondence}

Raj K. Shrivastava: Icahn School of Medicine at Mount Sinai, New York, NY. raj.shrivastava@mountsinai.org. 